\title{
Segmentação por Limiarização de Macroblocos e Data-filling Iterativo para Compressão de Documentos Compostos Utilizando o H.264
}

\author{
Alexandre Zaghetto e Ricardo L. de Queiroz
}

\begin{abstract}
Resumo-O padrão MRC (Mixed Raster Content) para compressão de documentos (ITU-T.44) especifica uma representação multicamada para documentos compostos. Espera-se que melhores taxas de compressão possam ser alcançadas quando algoritmos de pre-processamento e padrões de compressão mais eficientes são utilizados. $O$ presente artigo propõe a utilização de um algoritmo de segmentação por limiarização de macroblocos para decompor o documento original em três camadas (Foreground, Background e máscara). O preenchimento das regiões "irrelevantes" (data-filling) é realizado por meio de uma técnica iterativa baseda em wavelets. As camadas Background/Foreground são comprimidas com o H.264/AVC operando no modo INTRA e a máscara binária é codificada com o JBIG2. O resultado é um desempenho sem igual, como demonstrado pelos experimentos.
\end{abstract}

Palavras-Chave-H.264/AVC, JPEG-2000, Segmentação de página, Data-filling, Compressão de documentos, Mixed Raster Content.

Abstract-The Mixed Raster Content (MRC) ITU document compression standard (T.44) specifies a multi-layer representation of a compound document. It is expected that higher compression can be achieved if more efficient pre-processing and compression algorithms are used. In this paper we propose using a macroblock-based segmentation algorithm to decompose the original document into three layers (Foreground, Background and mask). The proposed framework also uses an iterative waveletbased data-filling algorithm. Background and Foreground layers are compressed using H.264/AVC operating in INTRA mode. JBIG2 is used to encode the binary mask. The result is an unrivaled performance for compressing compound documents as demonstrated by our experiments.

Keywords-H.264/AVC, JPEG-2000, Page segmentation, Datafilling, Document compression, Mixed Raster Content.

\section{INTRODUÇÃO}

O padrão MRC (Mixed Raster Content) para compressão de documentos (ITU-T.44) [1] especifica uma representação multicamada para documentos compostos. O presente artigo propõe a utilização de um algoritmo de segmentação por limiarização de macroblocos como parte do codificador MRC de três camadas baseado nos padrões H.264/AVC [8] e JBIG2 [19], apresentado em trabalho anterior [2]. O objetivo principal é apenas mostrar que tal codificador, aliado a técnicas de segmentação de página e preenchimento de regiões "irrelevantes" apropriadas, pode atingir melhores taxas de compressão que esquemas baseados em codificadores considerados o estado da arte em compressão de imagens estáticas.

Departamento de Engenharia Elétrica, Faculdade de Tecnologia, Universidade de Brasília, Brasília, DF. E-mails: zaghetto@image.unb.br, queiroz@ieee.org. Este trabalho foi parcialmente financiado pela HP Brasil.

\section{A. H.264/AVC-INTRA}

O mais novo padrão de codificação de video, o H.264/AVC [8], já foi amplamente descrito pela literatura [9][14]. Muitos artigos ilustraram seu desempenho, apresentando inúmeros resultados que o comparam a outros codificadores. Todos os resultados apontam para um aumento de desempenho de no mínimo o dobro em relação a outros padrões de compressão de video. A razão que o torna tão eficiente são pequenas melhorias que individualmente contribuem modestamente para o aumento substancial do ganho geral. Além desse aumento de desempenho, o AVC apresenta algumas vantagens adicionais.

O H.264/AVC é um padrão de compressão de video e não foi concebido para ser aplicado como uma ferramenta de compressão de imagens estáticas. Apesar disso, os vários avanços incorparados ao H.264/AVC não apenas estabelecem um novo patamar em compressão de video, mas também fazem dele um excelente codificador de imagens estáticas [15], [16]. Um desses avanços é o método de predição INTRA, o qual, combinado com o codificador aritmético binário adaptativo ao contexto, transforma o H.264/AVC em um poderoso compressor de imagens estáticas. Se o H.264/AVC for ajustado para codificar apenas um único quadro de video, ele se comporta como um codificador de imagens. Vamos nos referir a esse codificador como AVC-I. A grande surpresa é que o AVCI supera codificadores de imagens considerados o estado da arte, como o JPEG-2000 [17]. A surpresa advém do fato de tal codificador não ter sido inicialmente concebido para essa finalidade específica. Os ganhos do AVC-I sobre o JPEG-2000 tipicamente são da ordem de $0.25 \mathrm{~dB}$ a $0.5 \mathrm{~dB}$ em PSNR (peak signal-to-noise ratio) para imagens pictoriais [15], [16], [18].

\section{B. JBIG2}

O JBIG2 [19] é um padrão internacional para compressão de imagens binárias desenvolvido pelo Joint Bi-level Image Experts Group. Publicado em 2000 sob a denominação ITU-T Recommendation T.88, e em 2001 como ISO/IEC Standard 14492, o JBIG2 supera o JBIG1 [20] e o Group 4/ MMR [21] por um fator de 2 e 3, respectivamente [22]. Tipicamente, um codificador JBIG2 decompõe uma imagem binária em regiões (texto, halftone e genérica) e codifica cada região utilizando métodos diferentes.

\section{Documentos Compostos}

Documentos eletrônicos são basicamente representados de duas maneiras: vetorial e raster [4]. Não constitui grande 


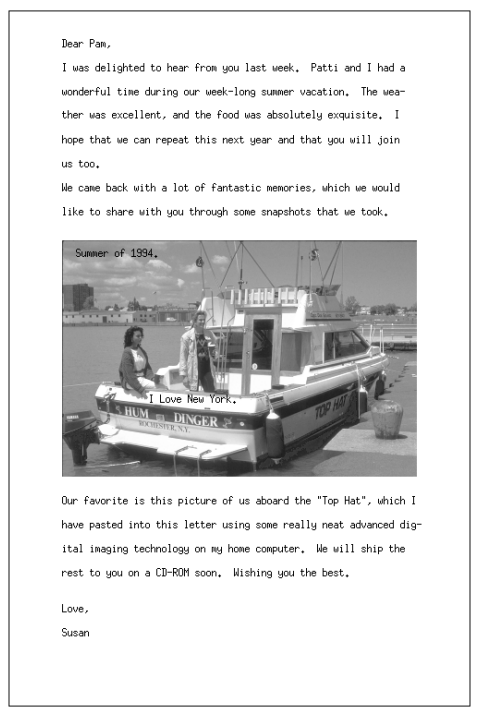

Fig. 1. "Compound1": exemplo de documento composto, considerado como sendo um documento representado na forma raster e que contém um mistura de texto e imagem.

desafio comprimir documentos vetorizados, uma vez que cada objeto pode ser comprimido individualmente e o arquivo todo pode ser comprimido sem perdas. O real desafio é comprimir documentos na forma raster. Documentos compostos serão considerados aqueles que são representados na forma raster e que contêm um mistura de texto e imagem.

Os algoritmos de compressão são desenvolvidos tendose em mente um tipo particular de imagem e aplicação. Nenhum algoritmo é o mais adequado para todos os tipos de imagens e aplicações. Quando se comprime texto, é importante preservar as bordas e os formatos dos caracteres para que a leitura seja facilitada. O sistema visual humano, por outro lado, trabalha diferentemente para imagens em tons contínuos, melhor mascarando erros de alta freqüência [23].

A Fig. 1 mostra um exemplo de documento composto.

\section{Mixed Raster Content}

Documentos compostos são tipicamente codificados como uma imagem única. No entanto, diferentes algoritmos de compressão podem ser aplicados a cada região do documento. É assim que um algoritmo baseado em múltiplos codificadores funciona. O modelo MRC (Mixed Raster Content) básico representa um documento por meio de duas camadas de imagem (Foreground ou FG e Background ou BG) e uma máscara binária, que determina se um pixel pertence ao FG ou BG [1][7]. A Fig. 2 ilustra o modelo descrito. Após a decomposição do documento, cada camada pode ser processada e comprimida por meio de algoritmos específicos. O processamendo do FG e do BG pode incluir mudança de resolução e data-filling. $\mathrm{O}$ algoritmo de compressão utilizado para uma determinada camada deve ser adequado ao seu conteúdo, possibilitando o aumento da compressão enquanto a distorção é reduzida. Após codificadas, as camadas são empacotadas e enviadas ao decodicador. No decodificador a imagem é recomposta por meio do processo inverso.

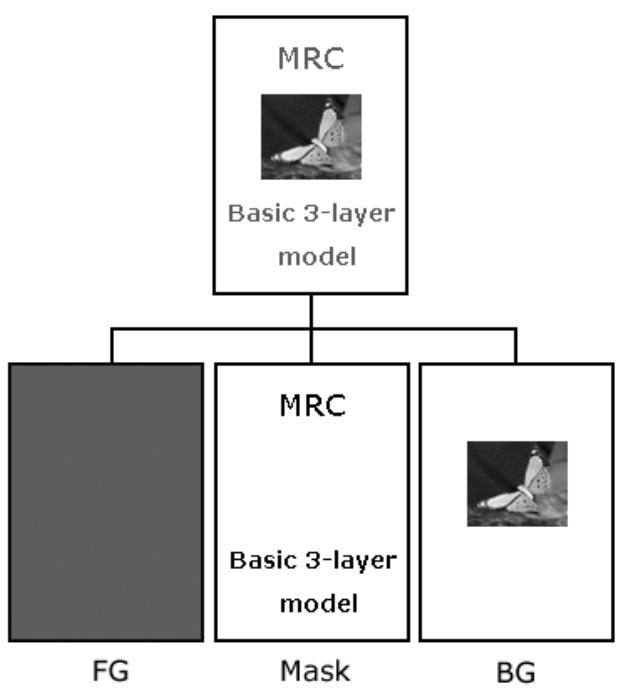

Fig. 2. Ilustração do modelo de imageamento MRC. O documento original é representado utilizando-se três camadas: Foreground (FG), Background (BG) e Máscara.

\section{SegmentaÇÃo}

O primeiro passo da compressão MRC é a segmentação [4]. No presente artigo, consideramos o modelo MRC básico de três camadas, que utiliza uma representação baseada em FG, BG e máscara. O algoritmo de segmentação descrito a seguir é uma variação do algoritmo baseado em limiarização por blocos proposto por De Queiroz, Fan e Tran [24].

Como o FG e o BG serão codificados por macroblocos (blocos de 16x16 pixels), desejamos encontrar uma máscara de macrobloco $m_{n}(i, j)$, para cada um dos $n$ macroblocos do documento. Devido ao fato de áreas que contêm bordas de texto normalmente apresentarem histogramas bimodais, uma abordagem simplificada seria encontrar os macroblocos bimodais e agrupar seus pixels ao redor das duas modas. Independentemente do método utilizado para se testar a bimodalidade ou realizar o agrupamento, os pixels são divididos por algum tipo de limiarização.

Na limiarização por macrobloco, a máscada $m_{n}(i, j)$ é dada por,

$$
m_{n}(i, j)=u\left(t_{n}-x_{n}(i, j)-1\right),
$$

onde $t_{n}$ é o limiar do bloco, $x_{n}(i, j)$ representa o macrobloco original e $u(k)$ é a função degrau discreta (igual a 1 para $k \geq 0$ e 0 caso contrário).

Em um macrobloco há 256 pixels e, por isso, 256 é o número de possíveis limiares. Para cada macrobloco, seleciona-se um conjunto de $l \leq 256$ limiares $t_{n}$ organizados em ordem crescente e procura-se minimizar a seguinte função de custo,

$$
J=\alpha_{1} V_{B G}+\alpha_{2} V_{F G}+\alpha_{3} N_{t},
$$

onde $\alpha_{i}$ são pesos, e $V_{B G}$ e $V_{F G}$ são as variâncias dos pixels dos macroblocos do $\mathrm{BG}$ e do $\mathrm{FG}$, respectivamente. $N_{t}$ é o número de transições horizontais de $m_{n}(i, j)$ (a primeira coluna da máscara do macrobloco atual é utilizada como 


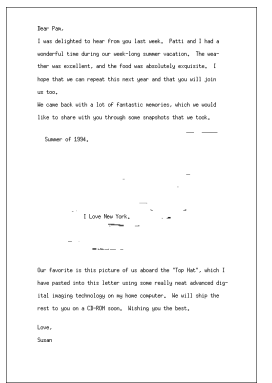

(a)

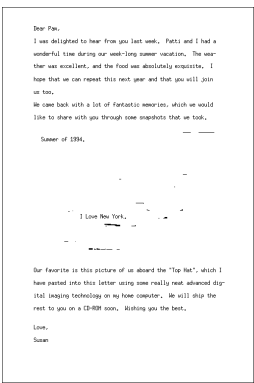

(b)

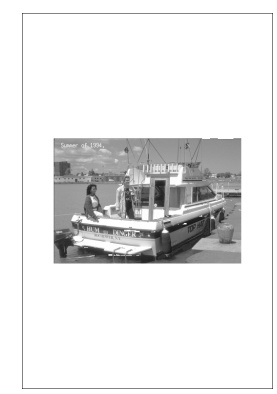

(c)
Fig. 3. Decomposição em camadas do documento "compound1" antes do procedimento de data-filling: (a) FG; (b) Máscara; (c) BG.

referência para a última coluna da máscara do macrobloco anterior). Valores de $V_{B G}$ e $V_{F G}$ mais elevados indicam uma menor compressão dos planos BG e FG, o que contribui para o aumento do custo. Em relação à máscara $m_{n}(i, j)$, quanto maior o número de transições $N_{t}$, menor a compressão, ou seja, maior o custo.

Para cada um dos $l$ limiares $t_{n}$, obtem-se $m_{n}(i, j)$, e dois conjuntos são definidos,

$$
\begin{aligned}
& X_{F G} \equiv\left\{x_{n}(i, j) \mid m_{n}(i, j)=0\right\} \\
& X_{B G} \equiv\left\{x_{n}(i, j) \mid m_{n}(i, j)=1\right\} .
\end{aligned}
$$

Define-se $n_{F G}$ e $n_{B G}$ como o número de pixels dos conjuntos $X_{F G}$ e $X_{B G}$, respectivamente. Obviamente, $n_{F G}+n_{B G}=$ 256. As variâncias são calculadas pelas equações,

$$
\begin{gathered}
V_{F G}=\frac{\sum_{X_{F G}} x_{n}(i, j)^{2}}{n_{F G}}-\left(\frac{\sum_{X_{F G}} x_{n}(i, j)}{n_{F G}}\right)^{2} \\
V_{B G}=\frac{\sum_{X_{B G}} x_{n}(i, j)^{2}}{n_{B G}}-\left(\frac{\sum_{X_{B G}} x_{n}(i, j)}{n_{B G}}\right)^{2}
\end{gathered}
$$

as quais podem ser implementadas de forma eficiente. Quanto aos pesos $\alpha_{i}$, sem perda de generalidade, pode-se normalizar um deles (p. ex., $\alpha_{2}=1$ ). A escolha dos outros dois pesos é realizada de forma empírica.

O limiar $t_{n}$ que resultar no menor custo $J$ é escolhido, a máscara $m_{n}(i, j)$ é definida e a separação em camadas para o macrobloco é realizada. Tal procedimento é repetido para cada um dos $n$ macroblocos do documento.

A Fig. 3 mostra a decomposição em camadas do documento "compound1", antes do procedimento de data-filling.

\section{DATA-FILLING}

Após a decomposição em camadas, pixels associados ao BG serão marcados como "irrelevantes" no FG. Da mesma forma, pixels associados ao FG serão marcados como "irrelevantes" no BG. Esses pixels podem ser substituidos por qualquer valor com o objetivo de se melhorar a compressão [4], [25], [26]. Existem vários métodos destinados a esse fim. O presente artigo utiliza um método de preenchimento iterativo baseado em wavelets [27], que será descrito a seguir.

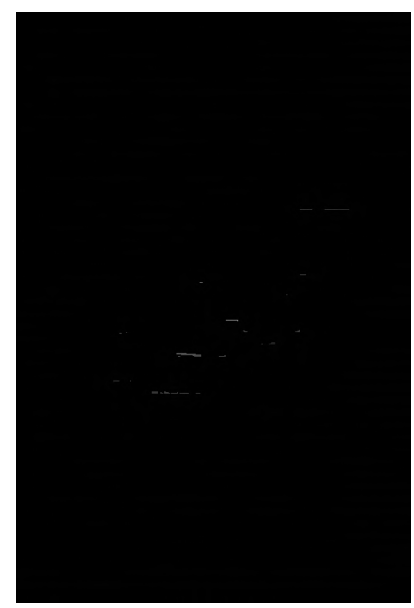

(a)

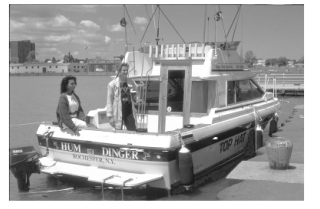

(b)
Fig. 4. (a) FG e (b) BG do documento "compound1" após o procedimento de data-filling.

Definiremos $F$ e $B$ como sendo as posições dos pixels onde a máscara do documento indica $\mathrm{FG}$ e $\mathrm{BG}$, respectivamente. Inicialmente, calcula-se as médias,

$$
\begin{aligned}
& m_{B G}=\operatorname{mean}(x(i, j) \mid(i, j) \in B) \\
& m_{F G}=\operatorname{mean}(x(i, j) \mid(i, j) \in F),
\end{aligned}
$$

onde $x(i, j)$ representa a imagem original.

Seja $I_{0}$ a camada FG inicial com os pixels "irrelevantes" substituidos por $m_{F G}$. Seja $\tilde{I_{n}}$ a versão codificada e decodificada de $I_{n}$, utilizando-se um codificador a uma dada taxa de bits alvo. Se planejamos utilizar um codificador baseado em wavelets, $\tilde{I}_{n}$ pode ser aproximado por,

$$
\tilde{I_{n}}=W^{-1}\left(\operatorname{round}\left(W\left(I_{n}\right) / Q\right) * Q\right),
$$

onde $W$ denota a Tranformada Wavelet, round(.) é o operador de arredondamento e $Q$ é o passo de quatinzação dos coeficientes da tranformada. Então, para $n=0$ até $n=\nu$, onde $\nu$ limita o número de ciclos em 3 ou 4, computa-se,

$$
I_{n+1}(i, j)=\left\{\begin{array}{lll}
I_{n}(i, j) & : & (i, j) \in F \\
\tilde{I}_{n}(i, j) & : & (i, j) \in B .
\end{array}\right.
$$

O algoritmo é interrompido após $\nu$ ciclos ou quanto,

$$
\operatorname{mean}\left(\left|I_{n}(i, j)-I_{n-1}(i, j)\right|\right)<\xi, \quad(i, j) \in B,
$$

onde $\xi$ é um limiar de tolerância. Em outras palavras, o algoritmo é interrompido quando o preenchimento das regiões "irrelevantes" converge. O mesmo processo é aplicado ao BG, substituindo-se a notação do FG pela do BG, e vice-versa. A Fig. 4 mostra as camadas do documento "compound1" após o processo de data-filling descrito anteriormente.

A Fig. 5 mostra a ampliação de uma parte do BG, onde o efeito do algoritmo de data-filling pode ser obsevado em detalhes. 


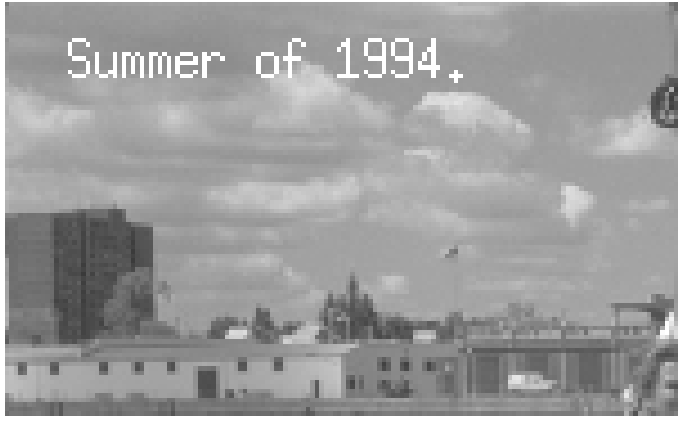

(a)

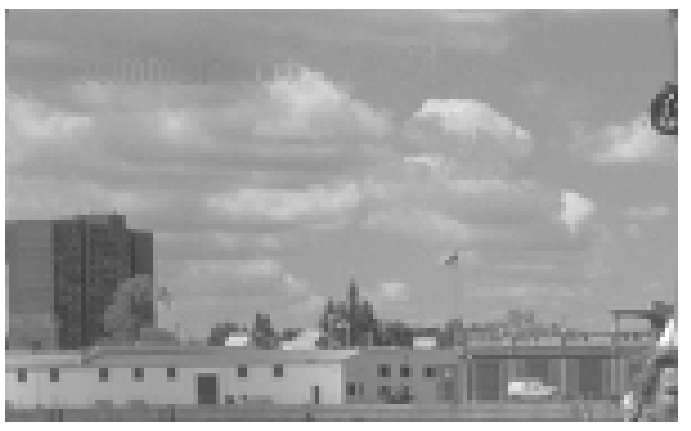

(b)

Fig. 5. Parte ampliada do BG do documento "compound1": (a) Documento original; (b) Após o data-filling.

\section{Resultados}

O documento "compound1" foi comprimido com os codificadores AVC-I, JPEG-2000 e o model MRC proposto. Na compressão MRC, a máscara foi codificada com o JBIG2 e as camadas BG/FG foram ambas codificadas com o JPEG-2000 e com o AVC-I. A Fig. 6 mostra as curvas PSNR resultantes.

Os documentos da Fig. 7 e 9 também foram comprimidos com os mesmos codificadores. Suas respectivas curvas PSNR podem ser observadas nas Figs. 8 e 10 .

O AVC-I parece ter uma capacidade extra em se adaptar a conteúdos heterodoxos [18]. Para os documentos compostos mostrados nas Figs. 1 e 7, por exemplo, os ganhos em PSNR são substânciais, ultrapassando a marca de $4 \mathrm{~dB}$ em relação ao JPEG-2000 a 1 bit/pixel. Apesar dessa capacidade extra do AVC-I, o model MRC proposto, baseado em múltiplos codificadores, fornece resultados que ultrapassam a abordagem que utiliza um codificador único em mais de $4 \mathrm{~dB}$ a 1 bit/pixel.

As curvas PSNR das Figs. 6, 8 e 10 também mostram que o modelo MRC baseado em AVC-I supera o modelo MRC baseado em JPEG-2000, um codificador considerado o estado da arte em compressão de imagens estáticas

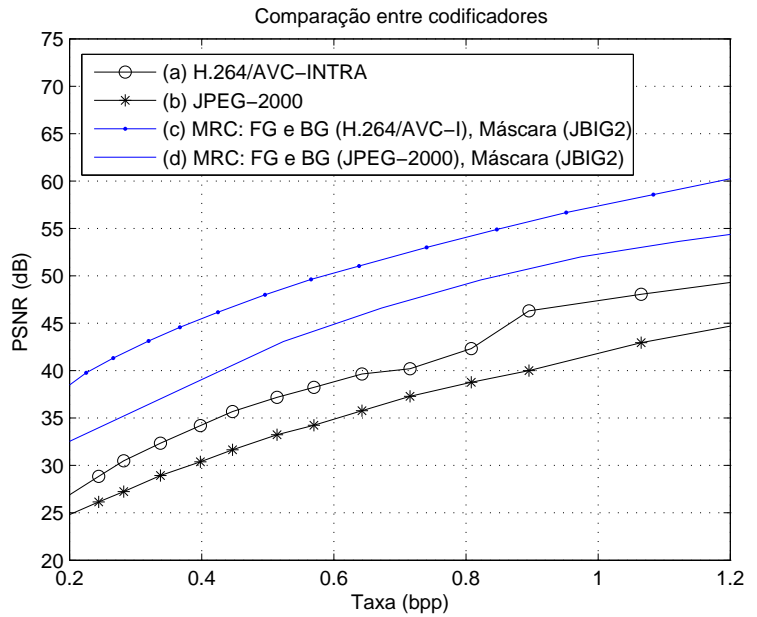

Fig. 6. Curvas PSNR para o documento "coumpound1", comparando: (a) AVC-I; (b) JPEG-2000; (c) MRC: FG e BG codificados com AVC-I e Máscara codificada com JBIG2; (d) MRC: FG e BG codificados com JPEG-2000 e Máscara codificada com JBIG2. A curvas mostram que o modelo MRC baseado em H.264/AVC-I supera o modelo MRC baseado em JPEG-2000 em mais de $4 \mathrm{~dB}$ a 1 bit/pixel. Pesos considerados: $\alpha_{1}=100, \alpha_{2}=1, \alpha_{3}=40$.

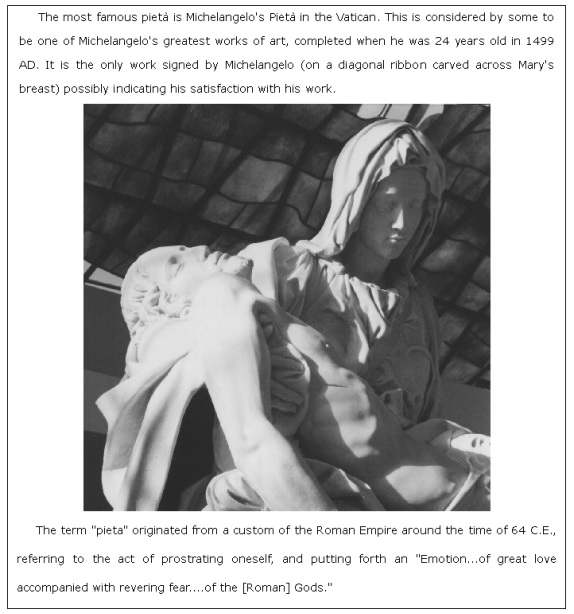

Fig. 7. "Pieta": exemplo de documento composto.

\section{CONClusões}

No presente artigo apresentamos um codificador MRC que utiliza o H.264/AVC operando no modo INTRA para codificar as camadas BG/FG e o JBIG2 para codificar a máscara binária. Também propomos a utilização de um algoritmo de segmentação baseado em limiarização de macrobloco e um algoritmo de preenchimento de regiões "irrelevantes". Os resultados mostram que com o modelo MRC é possível alcançar melhor desempenho do que as aborgagens baseadas em um codificador único, como o JPEG-2000 e o AVCI. Além disso, usar o AVC-I para codificar o BG e o FG em um codificador MRC resulta em melhor desempenho que esquemas que utilizam o JPEG-2000.

Sem dúvida, a proposta do modelo MRC baseado em AVCI, aliado às tecnicas de segmentação de página e data-filling apresentadas, estabelece um novo patamar para compressão de documentos compostos. 


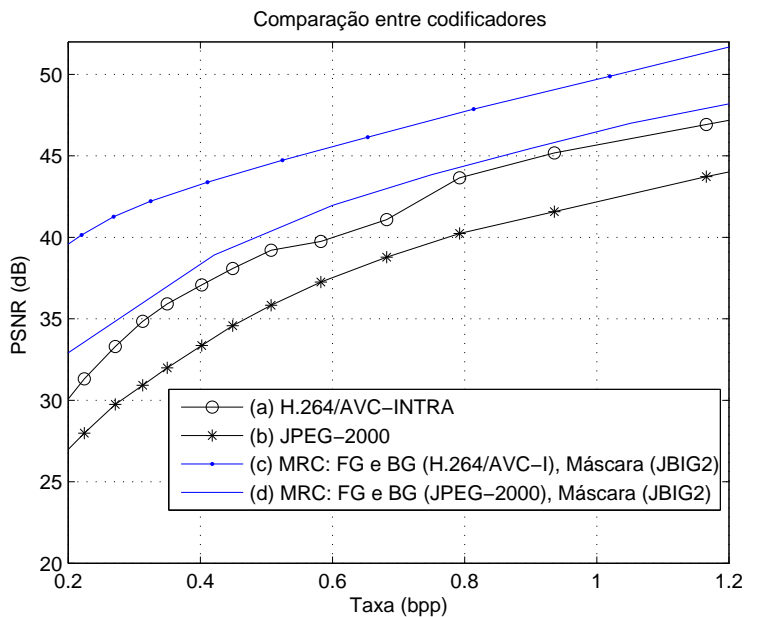

Fig. 8. Curvas PSNR para o documento "Pietà", comparando: (a) AVC-I; (b) JPEG-2000; (c) MRC: FG e BG codificados com AVC-I e Máscara codificada com JBIG2; (d) MRC: FG e BG codificados com JPEG-2000 e Máscara codificada com JBIG2. A curvas mostram que o modelo MRC baseado em H.264/AVC-I supera o modelo MRC baseado em JPEG-2000 em mais de 4 dB a 1 bit/pixel. Pesos considerados: $\alpha_{1}=500, \alpha_{2}=1, \alpha_{3}=40$.

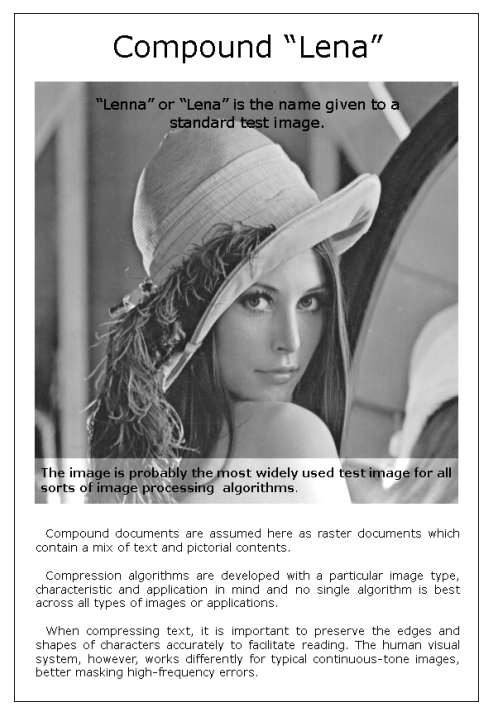

Fig. 9. "Compound Lena": exemplo de documento composto.

Apenas realizamos testes para imagens geradas por computador. Experimentos com imagens escaneadas estão sendo desenvolvidos.

\section{REFERÊNCIAS}

[1] "Mixed Raster Content (MRC)", ITU-T Recommendation T.44, 1999.

[2] A. Zaghetto and R. L de Queiroz, "MRC compression of compound documents using H.264/AVC-I", Simpósio Brasileiro de Telecomunicações, Recife, PE, Brasil, Set. 2007.

[3] R. L. de Queiroz, R. Buckley, M. Xu, "Mixed raster content (MRC) model for compound image compression", Proc. SPIE, Visual Communications and Image Processing, Vol. 3653, pp. 1106-1117, Jan 1999.

[4] R. L. de Queiroz, "Compressing Compound Documents", in The Document and Image Compression Handbook, edited by M. Barni, MarcelDekker, 2005

[5] D. Mukherjee, N. Memon, A. Said, , "JPEG-matched MRC Compression of Compound Documents", Proc. IEEE Intl. Conf. on Image Processing, ICIP, Vol. 3, Thessaloniki, Greece, pp. 434-437, Oct. 2001.

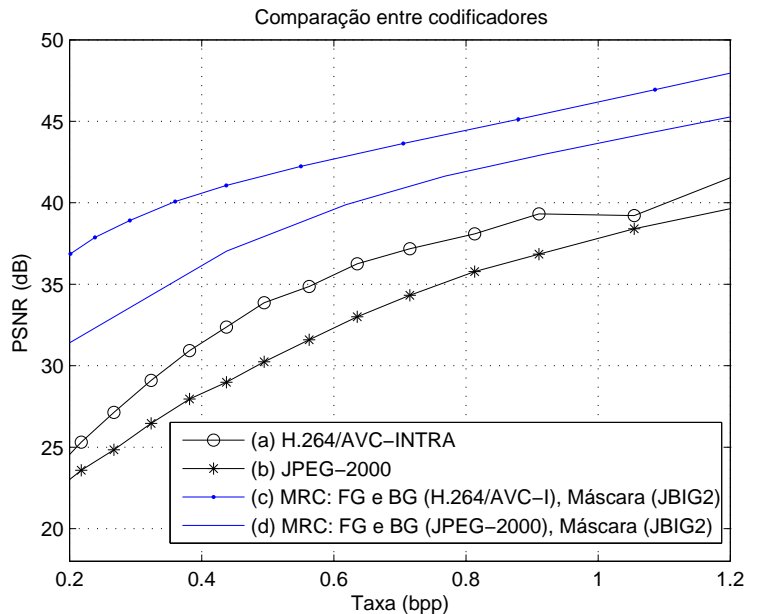

Fig. 10. Curvas PSNR para o documento "Compound Lena", comparando: (a) AVC-I; (b) JPEG-2000; (c) MRC: FG e BG codificados com AVC-I e Máscara codificada com JBIG2; (d) MRC: FG e BG codificados com JPEG2000 e Máscara codificada com JBIG2. A curvas mostram que o modelo MRC baseado em H.264/AVC-I supera o modelo MRC baseado em JPEG-2000 em mais de $3 \mathrm{~dB}$ a 1 bit/pixel. Pesos considerados: $\alpha_{1}=300, \alpha_{2}=1, \alpha_{3}=40$.

[6] D. Mukherjee, C. Chrysafis, A. Said, "JPEG2000-matched MRC compression of compound documents", Proc. IEEE Intl. Conf. on Image Processing, ICIP, Vol. 3, pp. 73-76, 2002.

[7] G. Feng and C.A. Bouman, "High-quality MRC document coding", IEEE Trans. on Image Processing, Vol. 15, No. 10, pp. 3152-3169, Oct. 2006.

[8] "Final Draft International Standard of Joint Video Specification", ITU-T Recommendation H.264, ISO/IEC 14496-10 AVC, March 2003.

[9] I. E. G. Richardson, H.264 and MPEG-4 Video Compression, Wiley, 2003.

[10] T. Wiegand, G. J. Sullivan, G. Bjontegaard, and A. Luthra, "Overview of the H.264/AVC video coding standard", IEEE Trans. on Circuits and Systems for Video Technology, Vol. 13, No. 7, pp. 560-576, July 2003.

[11] T. Stockhammer, M. M. Hannuksela, and T. Wiegand, "H.264/AVC in wireless environments", IEEE Trans. on Circuits and Systems for Video Technology, Vol. 13, No. 7, pp. 657-673, July 2003.

[12] T. Wiegand, H. Schwarz, A. Joch, F. Kossentini, and G. J. Sullivan, "Rate-constrained coder control and comparison of video coding standards", IEEE Trans. on Circuits and Systems for Video Technology, Vol. 13, No. 7, pp. 688-703, July 2003.

[13] G. J. Sullivan,P. Topiwala, and A. Luthra, "The H.264/AVC Advanced Video Coding Standard: Overview and Introduction to the Fidelity Range Extensions", Proc. of SPIE Conference on Applications of Digital Image Processing XXVII, Special Session on Advances in the New Emerging Standard: H.264/AVC, August, 2004.

[14] J. Ostermann, J. Bormans, P. List, D. Marpe, M. Narroschke, F. Pereira, T. Stockhammer, and T. Wedi, "Video coding with H.264/AVC: tools, performance, and complexity", IEEE Circuits and Systems Magazine, Vol. 4, No. 1, pp. 7-28, March 2004.

[15] D. Marpe, V. George, and T. Wiegand, "Performance comparison of intra-only H.264/AVC and JPEG2000 for a set of monochrome ISO/IEC test images", Contribution JVT ISO/IEC MPEG and ITU-T VCEG, JVT M-014, Oct. 2004.

[16] D. Marpe, V. George, H. L. Cycon, and K. U. Barthel, "Performance evaluation of Motion-JPEG2000 in comparison with H.264/AVC operated in pure intra coding mode", Wavelet Applications in Industrial Processing, in Proc. of SPIE, Vol. 5266, pp. 129-137, 2004.

[17] D. S. Taubman and M. W. Marcellin, JPEG2000: Image Compression Fundamentals, Standards, and Practice, Kluwer Academic, 2002.

[18] R. L. de Queiroz, R. S. Ortis, A. Zaghetto, and T. A. Fonseca, "Fringe benefits of the H.264/AVC", Proc. of Intl. Telecom. Symp., Fortaleza, Brazil, pp. 208-212, Sep. 2006.

[19] "Information technology - Coded representation of picture and audio information - Lossy/lossless coding of bi-level images", ITU-T Recommendation T.88, March 2000.

[20] "Information technology - Coded representation of picture and audio 
XXVI SIMPÓSIO BRASILEIRO DE TELECOMUNICAÇÕES - SBrT’08, 02-05 DE SETEMBRO DE 2008, RIO DE JANEIRO, RJ

information - Progressive bi-level image compression", ITU-T Recommendation T.82, March 1995.

[21] "Facsimile coding schemes and coding control functions for Group 4 facsimile apparatus", ITU-T Recommendation T.6, Nov. 1988.

[22] "JBIG Maui Meeting Press Release: New document compression standard quadruples compression of today's fax standards and runs at unprecedented speeds", ISO/IEC JTC1/SC29/WG1, Dec. 1999.

[23] D. Marr, Vision, San Francisco, CA: Freeman, 1982.

[24] R. L. de Queiroz, Zhigang Fan, T. D. Tran, "Optimizing blockthresholding segmentation for multilayer compression of compound images", IEEE Trans. on Image Processing, Vol. 9, No. 9, pp. 14611471, Sep. 2000.

[25] R. L. de Queiroz, "On data-flling algorithms for MRC layers", Proc. IEEE Intl. Conf. on Image Processing, ICIP, Vancouver, Canada, Vol. II, pp. 586-589, Sep. 2000.

[26] G. Lakhani, R. Subedi, "Optimal filling of FG/BG layers of compound document images", Proc. IEEE Intl. Conf. on Image Processing, ICIP, Atlanta, USA, pp. 2273-2276, , Oct. 2006.

[27] R. L. de Queiroz, "Pre-processing for MRC layers of scanned images", Proc. IEEE Intl. Conf. on Image Processing, ICIP, Atlanta, USA, pp. 3093-3096, , Oct. 2006. 